Ancin, B.L., Epherra, L. \& Rubilar, T. (2021). Efecto de la temperatura sobre la morfología y reproducción en el erizo de mar Arbacia dufresnii (Echinodermata: Echinoidea). Revista de Biología Tropical, 69(S1), 154-170. DOI 10.15517/rbt.v69iSuppl.1.46346

DOI 10.15517/rbt.v69iSuppl.1.46346

\title{
Efecto de la temperatura sobre la morfología y reproducción en el erizo de mar Arbacia dufresnii (Echinodermata: Echinoidea)
}

\author{
Barbara Luz Ancin ${ }^{1}$ \\ Lucía Epherra ${ }^{2 *}$ \\ Tamara Rubilar ${ }^{2,3}$
}

1. Universidad Nacional de la Patagonia San Juan Bosco. Puerto Madryn, Chubut, Argentina; barbiancin@yahoo.com.ar

2. Laboratorio de Oceanografia Biológica (LOBio), Centro para el Estudio de Sistemas Marinos, Centro Nacional Patagónico, Centro Científico Tecnológico del Consejo Nacional de Investigaciones Científicas y Técnicas (CESIMAR, CCT CENPAT, CONICET). epherra@cenpat-conicet.gob.ar (*Correspondence).

rubilar@cenpat-conicet.gob.ar

3. Laboratorio de Química de Organismos Marinos (LabQuiOM), Instituto Patagónico del Mar (IPAM), Universidad Nacional de la Patagonia San Juan Bosco. Puerto Madryn, Argentina. crubilar@unpata.edu.ar

Recibido 25-VI-2020. Corregido 15-VIII-2020. Aceptado 16-X-2020.

\author{
ABSTRACT \\ Effect of temperature on morphology and reproduction of sea urchin, Arbacia dufresnii \\ (Echinodermata: Echinoidea)
}

\begin{abstract}
Introduction: The increase in atmospheric $\mathrm{CO}_{2}$ values and the consequent warming of seawater constitute stressors for marine ecosystems, renovating interest in echinoid physiology. The echinoid Arbacia dufresnii is a widely studied species with a wide distribution in the North Patagonian gulfs. Objective: Evaluate the effect of seawater temperature on its morphology and reproduction. Methods: Individuals were exposed for 10 weeks (April-June 2016) to three temperature treatments $\left(13{ }^{\circ} \mathrm{C}\right.$ control, $15{ }^{\circ} \mathrm{C}$ and $\left.17{ }^{\circ} \mathrm{C}\right)$ according to the current conditions of seawater and the expected values for the next century. At the end of the experiment, diameter and weight of the whole individual and of its internal organs were statistically and histologically analyzed. Results: Individuals exposed to higher temperatures died in greater numbers. The control, and the $17{ }^{\circ} \mathrm{C}$ treatment, had no changes in dry gonad and intestine weights. However, the $15{ }^{\circ} \mathrm{C}$ group had wider diameter of test and a higher dry weight of gonads and intestine. At this temperature, the gonadal stages for males were similar to the environment, but females had more advanced stages. Conclusions: Temperature of $15^{\circ} \mathrm{C}$ could be optimal for assimilating food and obtaining energy. Gonads and intestine would be showing changes in the short term, unlike the calcified organs (test and Aristotle's lantern).
\end{abstract}

Key words: echinoid; thermal experiment; gametogenesis; gonadal production; physiology.

El exceso de gases liberados a la atmósfera genera un progresivo aumento de la temperatura y de dióxido de carbono $\left(\mathrm{CO}_{2}\right)$, produciendo procesos globales de cambio climático. Estos procesos afectan a los sistemas naturales y humanos en todos los continentes y en los océanos (IPCC, 2014). Debido a que el calentamiento de los océanos genera cambios en la circulación oceánica, en los patrones climáticos regionales y en la distribución de las precipitaciones; ocurren cambios físicos en el ambiente y esto produce estrés en los 
ecosistemas (Przeslawski, Ahyong, Byrne, Woerheide \& Hutchings, 2008; Brierley \& Kingsford, 2009). El océano ha absorbido más de un $80 \%$ del calor incorporado al sistema climático $\mathrm{y}$, en consecuencia, la temperatura media del mar ha aumentado alrededor de 0.6 ${ }^{\circ} \mathrm{C}$ en los últimos 100 años (IPCC, 2013). Las predicciones plantean que para el año 2100 la temperatura superficial del mar se incremente de 0.3 a $4.8{ }^{\circ} \mathrm{C}$ (IPCC, 2013). Dado que las zonas costeras son muy vulnerables y que sus ecosistemas son especialmente sensibles a las presiones ambientales como los cambios en la temperatura (IPCC, 2014), es esperable que los invertebrados y sus ecosistemas se vean afectados de forma aguda como también crónica, con efectos que van desde subletales a letales y variando durante su ciclo de vida (Przeslawski et al., 2008).

La temperatura del agua es el principal factor que determina el óptimo funcionamiento del metabolismo de los invertebrados marinos (Hochachka \& Somero, 2002). En general, un aumento de temperatura afecta la asimilación de alimentos, el crecimiento (Kemp \& Britz, 2008; Gooding, Harley \& Tang, 2009), la respiración (McElroy, Nguyen \& Byrne, 2012), el desarrollo de las gónadas, reproducción (Olive, 1995; Bates, 2005) y la supervivencia en los invertebrados marinos (Sanford, 2002; Gooding et al., 2009; González et al., 2010; McElroy et al., 2012). A su vez, el aumento de las temperaturas del mar se ha relacionado con cambios en patrones temporales de desove en regiones templadas (Edwards \& Richardson, 2004; Lawrence \& Soame, 2004).

El estado fisiológico es el principal determinante de la tolerancia de una especie a la variabilidad y cambio ambiental. Durante las últimas décadas hubo un renovado interés en la fisiología de los equinodermos, principalmente en la adaptabilidad de algunas especies frente a los cambios previstos de diferentes parámetros ambientales marinos (McGaw, Clifford \& Goss, 2015). En el caso particular de estudios sobre el posible efecto del cambio climático sobre los equinoideos, se los ha utilizado para estudiar los efectos sobre procesos fisiológicos tales como la respiración, la alimentación, reproducción, fertilización y, principalmente, el desarrollo larvario (Byrne, Andrew, Worthington \& Brett, 1998; Kurihara \& Shirayama, 2004; Byrne et al., 2009; Dupont \& Thorndyke, 2009; Byrne, 2010; Cohen-Rengifo, García, Hernández, Hernández \& Clemente, 2013; Wangensteen, Turon, Casso \& Palacín, 2013; Delorme \& Sewell, 2016). Los equinoideos suelen tener un rango óptimo de temperatura en el cual se desenvuelven apropiadamente en cuanto a los procesos fisiológicos (Hochachka \& Somero, 2002; Siikavuopio, Mortensen \& Christiansen, 2008; Gooding et al., 2009; Azad, Pearce \& McKinley, 2011; Watts, Hofer, Desmond, Lawrence \& Lawrence, 2011; Siikavuopio et al., 2012). Sin embargo, cuando los animales están expuestos a temperaturas fuera de ese rango, procesos físiológicos como la alimentación, el crecimiento, el desarrollo de las gónadas y/o la supervivencia pueden verse comprometidos negativamente (Siikavuopio et al., 2008; Lawrence et al., 2009; Zamora \& Jeffs, 2012; Uthicke, Liddy, Nguyen \& Byrne, 2014). Los cambios en el metabolismo pueden afectar de forma diferente las distintas funciones fisiológicas, así, en erizos de mar, tanto la linterna de Aristóteles (órgano masticador) como el caparazón (endoesqueleto) suelen mostrar respuestas a cambios ambientales de forma gradual (Lawrence \& Ellwood, 1991), mientras que los órganos internos, de metabolismo rápido, como las gónadas y el tracto digestivo, lo hacen a escalas de tiempo cortas (días a semanas) (Russell, 1998).

La gran mayoría de las especies de equinoideos son herbívoros y cumplen funciones claves en los ecosistemas costeros que habitan, siendo capaces de alterar la abundancia, distribución y composición de las asociaciones de algas e invertebrados (Pérez, Gil \& Rubilar, 2014). Por lo tanto, cambios a nivel fisiológico individual con implicancias en la supervivencia y la reproducción de la población (Delorme \& Sewell, 2016) pueden tener consecuencia también a nivel de ecosistema (Paine, 1966; Harrold \& Pearse, 1987; Hernández, Bückle, Guisado, Barón \& Estavillo, 2004). El erizo 
de mar Arbacia dufresnii presenta una amplia distribución a lo largo de la costa Argentina, siendo una de las especies dominantes en las comunidades bentónicas de los golfos norpatagónicos (Bernasconi, 1953; Brogger et al., 2013; Epherra, 2016). Estudios en la población de $A$. dufresnii de Golfo Nuevo (Patagonia, Argentina) han mostrado que en años más cálidos se observa una mayor producción gonadal y un periodo de desove más prolongado (Epherra et al., 2015). Por otro lado, estudios en laboratorio mostraron que el desarrollo embrionario y larval se ven afectados significativamente por un aumento de la temperatura (Fernández, Chaar, Epherra, González-Aravena \& Rubilar, 2021). Estos antecedentes fueron claves para plantear el estudio en condiciones controladas del efecto del aumento de la temperatura del agua de mar sobre parámetros morfológicos y reproductivos del erizo de mar Arbacia dufresnii (Echinodermata: Echinoidea).

\section{MATERIALES Y MÉTODOS}

Muestreo: Se recolectaron 87 individuos adultos de Arbacia dufresnii (abril de 2016) mediante buceo autónomo en Punta Cuevas (Golfo Nuevo, 4246’3” S \& 6454'51" W); los organismos fueron transportados al Servicio de Acuario Experimental del CCT CENPAT donde fueron aclimatados durante una semana en un acuario de 301 a $14{ }^{\circ} \mathrm{C}( \pm 1)$, salinidad 33-34 ppm y un fotoperiodo de 12:12. Durante este tiempo, los erizos de mar no fueron alimentados con el objetivo de comenzar el experimento con todos los organismos en la misma condición.

Diseño experimental: Previo al comienzo del experimento, se individualizaron los organismos, se determinó el peso sumergido utilizando una balanza gravimétrica $(0.01 \mathrm{~g})$ y el diámetro máximo del caparazón mediante un calibre digital $(0.01 \mathrm{~mm})$ para cada uno. Se seleccionaron 15 individuos (ocho hembras y siete machos) al azar para establecer los parámetros a estudiar de la población natural (de ahora en más llamado "INICIO") antes de comenzar el experimento. Éstos fueron anestesiados con $\mathrm{Cl}_{2} \mathrm{Mg} 5 \%$ previamente a ser diseccionados siguiendo recomendaciones de Rubilar y Crespi-Abril (2017) para determinar el peso de los órganos: gónadas, intestino (conjunto de esófago, intestino y ano), caparazón y linterna de Aristóteles. Los pesos secos (balanza gravimétrica, $0.001 \mathrm{~g}$ ) se obtuvieron llevando los órganos hasta peso constante en estufa a $60{ }^{\circ} \mathrm{C}$.

Los 72 individuos restantes fueron utilizados para llevar a cabo el experimento que tuvo una duración de ocho semanas (65 días), abarcando parte del periodo de gametogénesis de A. dufresnii, que transcurre principalmente en otoño e invierno (Brogger et al., 2010; Epherra et al., 2015; Rubilar et al., 2016).

Se realizaron tres tratamientos utilizando como factor la temperatura del agua de mar: $13{ }^{\circ} \mathrm{C}( \pm 0.7), 15{ }^{\circ} \mathrm{C}( \pm 1.0)$ y $17{ }^{\circ} \mathrm{C}( \pm 0.7)$. De acuerdo con (Pisoni, Dellatorre \& Rivas, 2017), los valores promedio son $15.6{ }^{\circ} \mathrm{C}$ en abril, $13.9{ }^{\circ} \mathrm{C}$ en mayo y $12.4{ }^{\circ} \mathrm{C}$ en junio, por lo que el tratamiento a $13{ }^{\circ} \mathrm{C}$ es posible considerarlo como control, puesto que oscila entre los valores naturales para la época de estudio, mientras los otros dos tratamientos a $15^{\circ} \mathrm{C}$ y 17 ${ }^{\circ} \mathrm{C}$ representan valores elevados para la época del año (Dellatorre, Pisoni, Barón \& Rivas, 2012; Pisoni et al., 2017). La segunda $\left(15^{\circ} \mathrm{C}\right)$ representa un incremento de dos unidades por encima del control, el aumento intermedio esperado para fines del siglo XXI y la última $\left(17{ }^{\circ} \mathrm{C}\right)$ representa una temperatura extrema que podría llegar a darse de acuerdo con los modelos máximos calculados en zonas costeras para finales del siglo XXI $\left(+4{ }^{\circ} \mathrm{C}\right)$, de no mediar la disminución de emisiones de dióxido de carbono $\left(\mathrm{CO}_{2}\right)$ a la atmósfera (IPCC, 2014).

En cada tratamiento se utilizaron 24 individuos, separados individualmente para evitar la competencia por espacio y alimento. Cada acuario contenía 31 de agua de mar filtrada (300 micras y filtro UV), un aireador y un filtro de canasta (biológico y mecánico) que permite asegurar la oxigenación y la calidad del agua. Se colocaron de a seis acuarios individuales dentro de un recipiente de plástico de mayor 
tamaño, con agua de mar y un calentador (Atman) asociado a un sensor de temperatura programado a la temperatura deseada. La salinidad del agua se mantuvo entre 33-34 ppm con un fotoperiodo de 12:12. Tanto la temperatura como la salinidad fueron monitoreadas y registradas diariamente. Previo al comienzo del experimento, se corroboró que no exista diferencia en el diámetro y peso sumergido (ANOVA, $\mathrm{P}=0.96$ y $\mathrm{P}=0.46$, respectivamente) entre tratamientos.

Durante el experimento, los ejemplares fueron alimentados ad libitum dos veces por semana con similar cantidad de alimento artificial (aprox. $400 \mathrm{mg}$, Texas A\&M AgriLife Research) de acuerdo a Rubilar et al. (2016). Previamente a ser alimentados, se retiraron los restos de alimento no consumidos y las heces producidas $\mathrm{y}$, a su vez, se realizaba un recambio de dos tercios del agua de mar filtrada en cada acuario, mediante succión del fondo con una manguera, para evitar acumulación de compuestos nitrogenados. Se monitoreó el estado de los individuos mediante la observación de pérdida de espinas (presencia de espinas en el fondo), desoves (color del agua), posición y movilidad de los individuos y mortalidad. Si un individuo moría durante el experimento se extraía inmediatamente.

Procesamiento de las muestras: Al finalizar el experimento, se determinó el peso sumergido utilizando una balanza gravimétrica (0.01 g), y el diámetro máximo del caparazón mediante un calibre digital $(0.01 \mathrm{~mm})$. Luego, los individuos fueron anestesiados con $\mathrm{Cl}_{2} \mathrm{Mg}$ $5 \%$ para ser diseccionados y así determinar el peso seco de los órganos (gónadas, intestino, caparazón y linterna de Aristóteles) de la misma forma que los individuos al inicio del experimento (INICIO). El sexo fue establecido por la coloración gonadal y análisis histológicos. Una porción de las gónadas fue fijada en Davidson por $24 \mathrm{~h}$ y conservada en etanol $70 \%$. El peso húmedo de esta porción fue registrado para luego corroborar el peso seco real de las gónadas de cada individuo. Para determinar los estadios gonadales se realizaron análisis histológicos de los tejidos de la gónada que se deshidrataron en una serie de alcoholes (70\%, $96 \%, 100 \%)$, luego se transparentaron los tejidos en xileno y, finalmente, fueron embebidos e incluidos en parafina. Los tejidos embebidos en parafina fueron seccionados cada $7 \mu \mathrm{m}$ con micrótomo rotacional Leica RM2255 y se tiñeron siguiendo la técnica de tinción con hematoxilina y eosina (Wilson \& Gamble, 2002). Los preparados se observaron en un microscopio óptico Carl Zeiss DM2500 y las fotografías se obtuvieron utilizando una cámara Leica DFC 280. Los estadios gonadales se clasificaron considerando las células germinales y los fagocitos nutritivos de acuerdo con Epherra et al. (2015).

Al igual que al comienzo del experimento, 15 individuos adultos del erizo de $\operatorname{mar} A$. dufresnii fueron recolectados de la población natural y procesados de la manera mencionada anteriormente, de ahora en más llamado FINAL. En este caso se hallaron siete hembras y ocho machos.

Para analizar el efecto de la temperatura y el sexo sobre los parámetros medidos (peso total sumergido, diámetro total del cuerpo, peso seco de las gónadas, intestino, linterna de Aristóteles y caparazón) se utilizaron Modelos Lineales Generalizados (GLM) (McCullagh, 1984). En primera instancia, se realizó una exploración de datos gráfica para comprender la relación entre la variable respuesta y cada una de las variables explicatorias. Los gráficos de caja (Box-plot) se utilizaron para variables discretas y permitieron eliminar variables explicatorias que estén altamente correlacionadas. Para comprobar el ajuste a los datos, se observó la cantidad de varianza explicada por el modelo, la devianza $\left(\mathrm{D}^{2}\right)$, la cual nos da una idea de la variabilidad de los datos. Los modelos fueron construidos del más simple (sin ningún factor) hasta el más complejo que incluye la interacción entre los factores (lo cual nos indica la dependencia de los factores), se analizaron y se seleccionó el mejor modelo con base en tres criterios: i) Criterio de información de Akaike (AIC del inglés Akaike Information Criterion, índice que evalúa tanto el ajuste del 
modelo a los datos como la complejidad del mismo); ii) Análisis de los residuos (análisis de los gráficos de residuos observados frente a estimados), y iii) Principio de parsimonia (el modelo debe ser tan simple como sea posible). En los resultados se muestran los valores AIC. Todos estos análisis fueron realizados con el software libre R (R Core Team, 2014).

Para analizar el efecto de la temperatura del agua de mar sobre las variables peso total y diámetro del cuerpo, así como el peso seco del caparazón de los erizos se utilizaron como variables respuestas la variación del diámetro del cuerpo (diámetro final-diámetro inicial), la variación del peso total del cuerpo (peso finalpeso inicial) y peso seco del caparazón al final del experimento; y como variables explicatorias, temperatura (niveles: $13{ }^{\circ} \mathrm{C}, 15{ }^{\circ} \mathrm{C}$ y 17 ${ }^{\circ} \mathrm{C}$ ) y sexo (niveles: machos y hembras). Para estudiar el efecto de la temperatura del agua de mar sobre las variables peso seco de gónada, de intestino, de la linterna de Aristóteles se seleccionaron como variables explicatorias, la temperatura (niveles: $13{ }^{\circ} \mathrm{C}, 15^{\circ} \mathrm{C}$ y $17^{\circ} \mathrm{C}$ ), sexo (niveles: macho y hembra); y a su vez se utilizó el diámetro final del cuerpo (DF) como covariable (variable explicatoria continua), ya que existe una relación alométrica entre el diámetro del caparazón y los órganos de los individuos (Ebert, Hernández \& Russell, 2011).

Para evaluar el efecto de la temperatura del agua de mar sobre la gametogénesis de los erizos, se realizó una descripción de los estadios gonadales. Mediante el análisis de la proporción en los cuales se encontraron en los distintos tratamientos de temperatura y en la población natural al inicio (INICIO) y final del experimento (FINAL). A diferencia de los objetivos anteriores en este se incorporan datos de la población natural.

\section{RESULTADOS}

La supervivencia en cada tratamiento se muestra en la Tabla 1. Cabe destacar que en el tratamiento de $17^{\circ} \mathrm{C}$, en un mismo día ocurrió la mortalidad de seis individuos (un cajón), los cuales mostraban signos de enfermedad ya que el agua presentaba turbidez y los organismos habían perdido espinas y se observaban signos de descomposición.

El modelo que presentó el mejor ajuste a los datos para variación del diámetro fue el que incluía el factor temperatura (Tabla 2), donde el tratamiento Temperatura $=15{ }^{\circ} \mathrm{C}$ presenta valores significativamente mayores respecto a las otras temperaturas (Fig. 1A, Tabla 3).

El mejor ajuste a los datos para variación del peso individual fue el modelo que incluía únicamente el factor sexo (Figura 1B, Tabla 2, Tabla 3). No se seleccionó el modelo nulo aunque la diferencia según el criterio de información de Akaike es muy baja, ya que al realizarle los estudios de los residuales el modelo seleccionado (Sexo) mostró mejor ajuste a los datos.

Los resultados de los tratamientos térmicos muestran que la temperatura y el sexo no son factores que expliquen la variación del peso seco del caparazón, puesto que el modelo

TABLA 1

Arbacia dufresnii. Porcentaje de supervivencia y número de individuos supervivientes al final del experimento separados por tratamiento de temperatura y sexo. $\mathrm{N}$ inicial $=24$ individuos por tratamiento

TABLE 1

Arbacia dufresnii. Survival percentage and number of surviving individuals at the end of the experiment separated by temperature treatment and sex. Initial $\mathrm{N}=24$ individuals per treatment

\begin{tabular}{|c|c|c|c|c|c|}
\hline \multicolumn{6}{|c|}{ Temperatura } \\
\hline \multicolumn{2}{|c|}{$13^{\circ} \mathrm{C}$} & \multicolumn{2}{|c|}{$15^{\circ} \mathrm{C}$} & \multicolumn{2}{|c|}{$17^{\circ} \mathrm{C}$} \\
\hline \multicolumn{2}{|c|}{$83.33 \%$} & \multicolumn{2}{|c|}{$66.66 \%$} & \multicolumn{2}{|c|}{$41.67 \%$} \\
\hline Machos & Hembras & Machos & Hembras & Machos & Hembras \\
\hline 10 & 10 & 4 & 12 & 5 & 5 \\
\hline
\end{tabular}


TABLA 2

Modelos propuestos para analizar la variable variación del diámetro (diámetro final- diámetro inicial), variación del peso individuo (peso final-peso inicial) y peso seco del caparazón. Resaltado en negrita se observa el modelo seleccionado que presentó mejor ajuste a los datos según Criterio de información de Akaike (AIC). La columna diferencia es el valor correspondiente a la diferencia del valor de AIC entre el modelo nulo y los distintos modelos

TABLE 2

Models proposed to analyze the variable variation of diameter (final diameter - initial diameter) and variation of individual weight (final weight-initial weight). Highlighted in bold is the selected model that presented the best fit to the data according to the Akaike Information Criterion (AIC). The difference column is the value corresponding to the difference of the AIC value between the null model and the different models

\begin{tabular}{lcccccc}
\multicolumn{1}{c}{ Modelos } & \multicolumn{2}{c}{ Variación Diámetro } & \multicolumn{2}{c}{ Variación Peso } & \multicolumn{2}{c}{ Peso Seco Caparazón } \\
& AIC & Diferencia & AIC & Diferencia & AIC & Diferencia \\
Nulo & 170.327 & 4480.433 & 1562.645 & 0.1430111 & $\mathbf{8 5 . 9 4 1 4 7}$ & $\mathbf{0}$ \\
Temperatura & $\mathbf{1 6 5 . 8 4 6}$ & $\mathbf{0}$ & 1568.504 & 0.7289927 & 88.54975 & 2.60828 \\
Sexo & 172.284 & 6437.303 & $\mathbf{1 5 6 1 . 2 1 5}$ & $\mathbf{0}$ & 87.9413 & 1.9998269 \\
Temperatura + Sexo & 167.682 & 1833.493 & 1577.621 & 16406.828 & 90.47738 & 4.5349087 \\
Temperatura * Sexo & 170.410 & 4563.677 & 1611.107 & 49891.988 & 86.52121 & 0.5797337 \\
\hline
\end{tabular}

TABLA 3

Parámetros de los modelos seleccionados para variables morfológicas de Arbacia dufresnii obtenidos mediante análisis de GLM. Resaltado en negrita se muestra la significancia en el tratamiento correspondiente.

Donde, $" * * * "=0, “ * * "=0,001, " * * "=0,01, " . "=0,05$. Int: intercepto

TABLE 3

Parameters of the selected models for morphological variables of Arbacia dufresnii obtained by GLM analysis.

Highlighted in bold, the significance of the corresponding treatment is shown.

Where $" * * * "=0, “ * * "=0.001, " * * "=0.01, " . "=0.05$. Int: intercept

\begin{tabular}{lccccc}
\multicolumn{1}{c}{ Variable } & Coeficientes & Estimado & Error Estándar & Valor t & $\operatorname{Pr}(>|\mathrm{t}|)$ \\
Variación de Diámetro & 13 (Int) & -0.0855 & 0.3112 & -0.275 & 0.7848 \\
& $\mathbf{1 5}$ & $\mathbf{1 . 3 5 7 4}$ & $\mathbf{0 . 4 6 6 8}$ & $\mathbf{2 . 9 0 8}$ & $\mathbf{0 . 0 0 5 7 * *}$ \\
\multirow{2}{*}{ Variación de Peso } & 17 & 0.8515 & 1.58 & 0.539 & 0.1215 \\
& Hembra (Int) & 1.5215 & 0.2675 & 5.689 & $<0.0001 * *$ \\
Peso Seco Caparazón & Macho & 1.3574 & 0.4668 & 2.908 & 0.0057 \\
\hline
\end{tabular}

seleccionado es el nulo (Figura 1C, Tabla 2, Tabla 3).

Respecto al análisis del peso seco de los órganos en los diferentes tratamientos térmicos, los modelos que mejor ajustan fueron diferentes según la variable analizada. En el peso seco de gónadas, tanto la temperatura como el sexo son los factores que mejor explican su variación entre tratamientos, el modelo seleccionado es el que presenta interacción entre los factores (Tabla 4). Los machos del tratamiento a $15{ }^{\circ} \mathrm{C}$ presentaron valores significativamente mayores $(\mathrm{P}<0.05)$ respecto a hembras y a las otras temperaturas (Figura 2A, Tabla 5). El modelo nulo presentó el mejor ajuste a los datos según el criterio de información de Akaike, pero en los estudios de los residuales, el modelo seleccionado (Sexo) mostró mejor ajuste a los datos.

Los resultados en peso seco de intestino muestran que la temperatura junto con la covariable DF son los factores que explican la diferencia, el modelo seleccionado es el que presenta aditividad entre éstos (Tabla 4). En 
TABLA 4

Modelos propuestos para analizar la variable peso seco de gónadas, peso seco de intestino y peso seco de la linterna de Aristóteles. Resaltado en negrita se observa el modelo seleccionado que presentó mejor ajuste a los datos según Criterio de información de Akaike (AIC). La columna diferencia es el valor correspondiente a la diferencia del valor de AIC entre el modelo nulo y los distintos modelos

TABLE 4

Models proposed to analyze the variable dry weight of gonads, dry weight of intestine and dry weight of Aristotle's lantern. Highlighted in bold is the selected model that presented the best fit to the data according to the Akaike Information Criterion (AIC). The difference column is the value corresponding to the difference of the AIC value between the null model and the different models

\begin{tabular}{lcccccc}
\multicolumn{1}{c}{ Modelos } & \multicolumn{2}{c}{ Peso seco Gónadas } & \multicolumn{2}{c}{ Peso seco Intestino } & \multicolumn{2}{c}{ Peso seco Linterna } \\
& AIC & Diferencia & AIC & Diferencia & AIC & Diferencia \\
Nulo & -1996.935 & 3413.937 & -8889.258 & 13339.512 & -8637.853 & 12984.153 \\
Temperatura & -1855.258 & 48.307 & -8832.319 & 13908.907 & -8258.012 & 16782.556 \\
Sexo & -2075.338 & 2629.904 & -8689.275 & 15339.351 & -857.519 & 13610.776 \\
Temperatura + DF & -1704.784 & 6335.438 & $\mathbf{1 0 2 2 . 3 2 1}$ & $\mathbf{0}$ & -9689.122 & 2471.456 \\
Sexo + DF & -1965.627 & 3727.016 & -1000.752 & 2156.887 & $\mathbf{- 9 9 3 6 . 2 6 8}$ & $\mathbf{0}$ \\
Temperatura + Sexo & -209.654 & 2417.881 & -8651.277 & 1571.933 & -8196.613 & 17396.548 \\
Temperatura * Sexo & $\mathbf{- 2 ~} \mathbf{3 3 8 . 3 2 8}$ & $\mathbf{0}$ & -8430.765 & 17924.448 & -8185.126 & 17511.416 \\
\hline
\end{tabular}

TABLA 5

Parámetros de los modelos seleccionados para los pesos secos de los diferentes órganos de Arbacia dufresnii obtenidos mediante análisis de GLM. Resaltado en negrita se muestra la significancia en el tratamiento correspondiente. Donde, “***” $=0, “ * * "=0,001, “ * ”=0,01, “ . "=0,05$. Int: intercepto. DF: diámetro final (covariable)

TABLE 5

Parameters of the selected models for the dry weights of the different organs of Arbacia dufresnii obtained by GLM analysis. Highlighted in bold, the significance of the corresponding treatment is shown. Where, "***” $=0$, “**” $=0.001$, “*” $=0.01, “ . "=0.05$. Int: intercept. DF: final diameter (covariate)

\begin{tabular}{lccccc}
\multicolumn{1}{c}{ Variable } & Coeficientes & Estimado & Error Estándar & Valor t & $\operatorname{Pr}(>|\mathrm{t}|)$ \\
Gónadas & Hembra, 13 (Int) & 0.1517 & 0.0543 & 2.796 & $0.0080^{* *}$ \\
& Macho & 0.0261 & 0.0767 & 0.34 & 0.7356 \\
& 15 & -0.0003 & 0.07346 & -0.004 & 0.9965 \\
& 17 & -0.0572 & 0.09397 & 0.608 & 0.5466 \\
& Macho, 15 & $\mathbf{0 . 2 9 2 8}$ & $\mathbf{0 . 1 2 5 3}$ & $\mathbf{2 . 3 3 7}$ & $\mathbf{0 . 0 2 4 7 *}$ \\
Intestino & Macho, 17 & 0.0161 & 0.1383 & 0.116 & 0.908 \\
& 13 (Int) & -0.3500 & 0.1362 & -2.570 & $0.0139 *$ \\
& 15 & 0.0484 & 0.0244 & 1.981 & 0.0543 \\
Linterna de Aristóteles & 17 & 0.0049 & 0.0292 & 0.171 & 0.865 \\
& DF & 0.01993 & 0.0048 & 4.17 & $0.0001 * * *$ \\
& Hembra (Int) & -0.2373 & 0.1448 & -1.639 & 0.1087 \\
& Macho & -0.0179 & 0.0233 & -0.766 & 0.448 \\
\hline
\end{tabular}

detalle, el modelo seleccionado mostró que la temperatura $15{ }^{\circ} \mathrm{C}$ resulta significativamente mayor respecto a los demás niveles (Figura 2B, Tabla 5).
Los resultados en peso seco de la linterna de Aristóteles muestran que, al igual que en los individuos de campo, el sexo es un factor importante puesto que el modelo seleccionado 

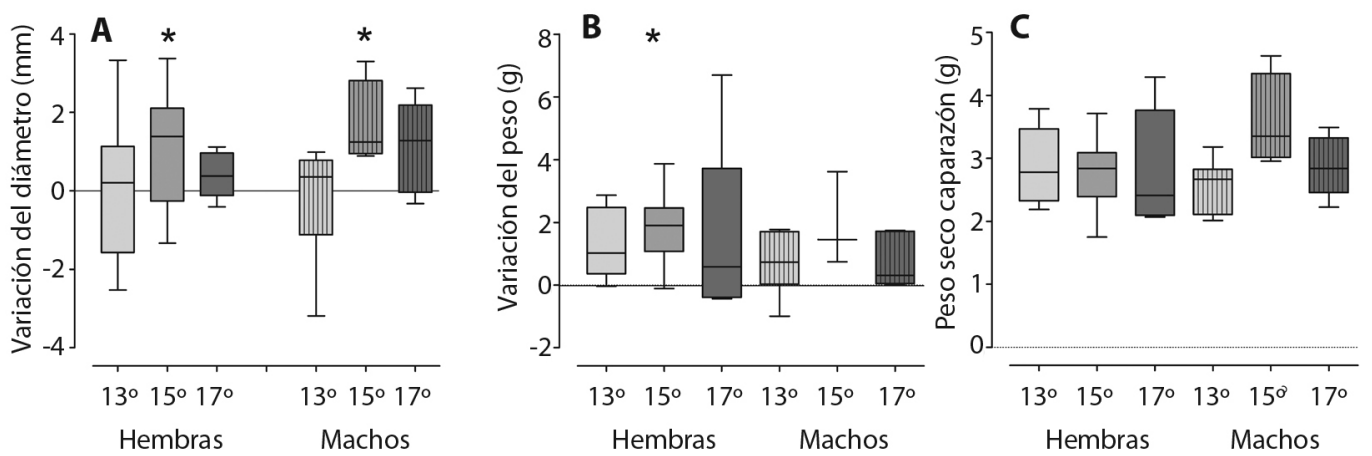

Fig. 1. Media, error estándar y rango de la variación del diámetro (a) y del peso del cuerpo (b); y el peso seco del caparazón (c) de Arbacia dufresnii para cada temperatura separados por sexo, los asteriscos (*) indican la diferencia significativa.

Fig. 1. Mean, standard error and range of variation in diameter (a) and body weight (b); and the dry weight of the shell (c) of Arbacia dufresnii for each temperature separated by sex, the asterisks $(*)$ indicate the significant difference.
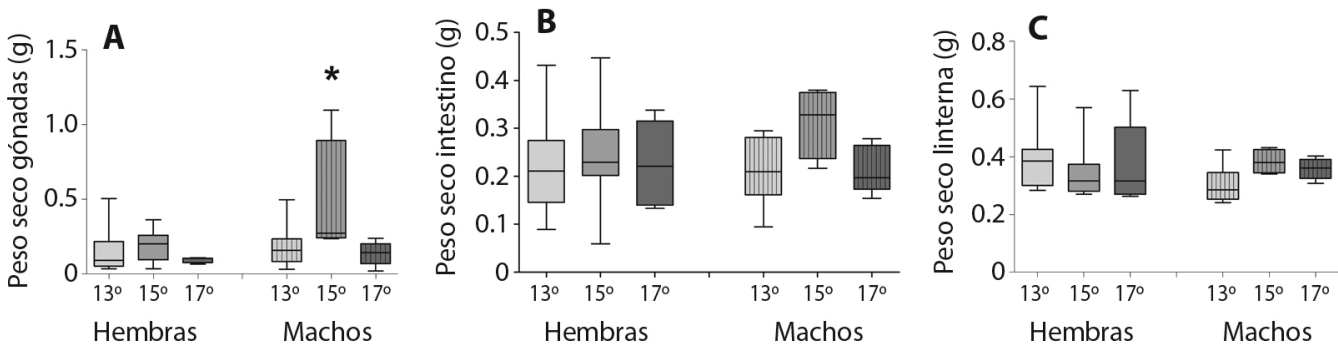

Fig. 2. Media, error estándar y rango del peso seco de gónadas (a.), peso seco de intestino (b.), y peso seco de linterna (c.) de Arbacia dufresnii para cada temperatura separados por sexo, los asteriscos $(*)$ indican la diferencias significativas.

Fig. 2. Mean, standard error and range of the dry weight of gonads (a.), Dry weight of intestine (b.), And dry weight of lantern (c.) of Arbacia dufresnii for each temperature separated by sex, the asterisks (*) indicate significant differences.

es el que presenta aditividad entre los factores sexo y diámetro final (Figura 2C, Tabla 4, Tabla 5).

Estadios gonadales del erizo de mar Arbacia dufresnii: Previo al comienzo del experimento, en ambos sexos los estadios gonadales de los erizos de mar provenientes de la población natural predominantes fueron pregamético e intergamético. Al final del experimento, en ambos sexos predominaron los estadios de crecimiento y premadurez. Los diferentes tratamientos térmicos presentaron mayor diversidad de estadios gonadales respecto a la población natural (Figura 3A, B).

En hembras, en el tratamiento de menor temperatura $\left(13^{\circ} \mathrm{C}\right)$ ningún estadio predominó sobre el otro, encontrándose individuos en estadio de crecimiento, premadurez, madurez e inclusive varios se encontraron desovados. Sin embargo, otros minoritarios no desarrollaron sus gónadas, pudiéndose observar un crecimiento nulo de las mismas. En el tratamiento de temperatura intermedia $\left(15^{\circ} \mathrm{C}\right)$, las hembras fueron más homogéneas, el mayor porcentaje de los individuos se encontraban en estadio de crecimiento y en estadio maduro, sólo unos pocos desovaron. En el tratamiento de temperatura elevada $\left(17^{\circ} \mathrm{C}\right)$ un gran porcentaje de los organismos se encontraban en fase de crecimiento y sólo unos pocos en premaduro y desove (Figura 3A).

Los machos en el tratamiento de menor temperatura $\left(13^{\circ} \mathrm{C}\right)$ presentaron predominancia 
A

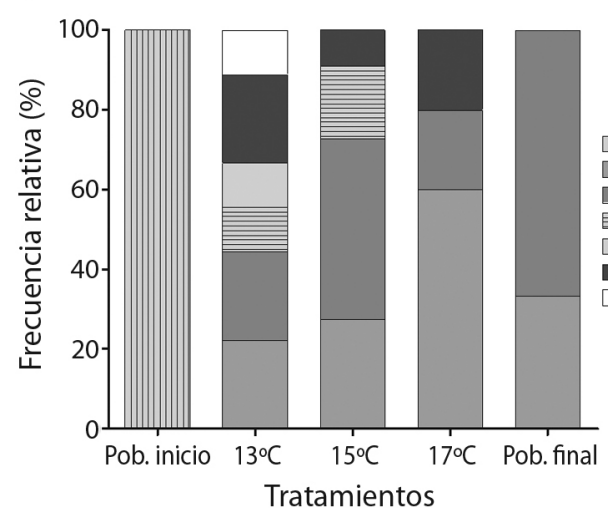

B

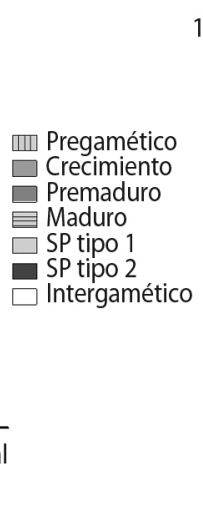

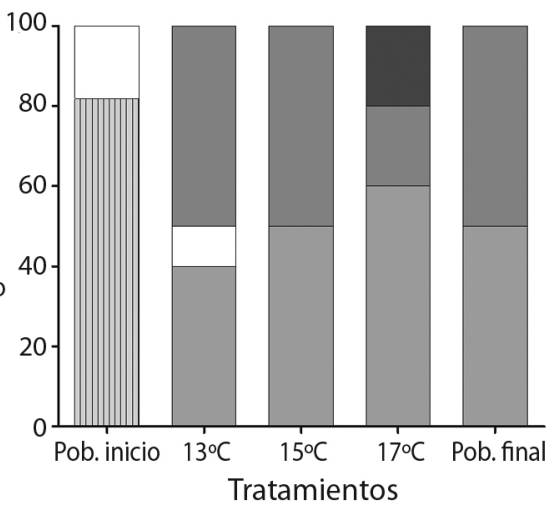

Fig. 3. Frecuencia relativa de los estadios gonadales de hembras (a.) y machos (b.) de Arbacia dufresnii en las diferentes temperaturas y en las poblaciones Inicio y Final.

Fig. 3. Relative frequency of the gonadal stages of females (a.) and males (b.) of Arbacia dufresnii at different temperatures and in the Start and End populations.

de solamente dos estadios, crecimiento y premaduros y en menor proporción intergaméticos. En el tratamiento de temperatura: $15{ }^{\circ} \mathrm{C}$ los estadios de los machos fueron homogéneos, la mitad se encontraban en el estadio premaduro y la otra mitad en crecimiento. En cambio, en el tratamiento de temperatura elevada (17 ${ }^{\circ} \mathrm{C}$ ) el mayor porcentaje de los organismos se encontraban en fase de crecimiento y sólo unos pocos en premaduro y desove al igual que las hembras (Figura 3B).

\section{DISCUSIÓN}

Los erizos de mar Arbacia dufresnii expuestos durante un período de ocho semanas a condiciones controladas con temperaturas mayores a su ambiente, mostraron variaciones en la morfología y reproducción respecto a individuos de la población natural. La temperatura influiría sobre diferentes parámetros fisiológicos de los organismos, expresados en variaciones en el diámetro, producción gonadal y peso del intestino. Sin embargo, el peso del organismo completo y de las estructuras calcificadas (linterna de Aristóteles y caparazón) no difirieron entre tratamientos. En Strongylocentrotus intermedius se encontraron resultados similares; es decir, no se observó efecto de la temperatura en individuos expuestos en tiempos de seis semanas a cuatro meses, sin embargo, sí se observó en individuos expuestos durante diez meses (Zhao et al., 2018). De esta forma, en el presente trabajo se corroboró que el tiempo de respuesta de los diferentes órganos depende del tiempo del estímulo y la función y naturaleza de este. Órganos como la linterna de Aristóteles y el caparazón suelen mostrar respuestas a cambios ambientales de forma gradual (Lawrence \& Ellwood, 1991), mientras que los órganos de metabolismo más rápido y de funciones de obtención y almacenamiento de energía (como gónadas e intestino) muestran respuestas más rápidas (Russell, 1998; Rubilar et al., 2016).

$\mathrm{Al}$ analizar la mortalidad durante el experimento, se observó que la temperatura afectaría el porcentaje de supervivencia dado que los tratamientos de mayor temperatura presentaron los valores más altos de mortalidad (duplicando al control). En otras especies como Strongylocentrotus franciscanus, Arbacia lixula, Heliocidaris erythrogramma, S. intermedius la mortalidad también es afectada por la temperatura en experimentos de laboratorio (Hernández et al., 2004; Harianto, Nguyen, Holmes \& Byrne, 2018; Sastre-Perelló, 2018; Zhao et al., 2018). En este trabajo, A. dufresnii 
presentó similar deterioro que el descripto por Sastre-Perelló (2018) en A. lixula expuesto a diferentes tratamientos de temperatura, donde los individuos parecen estar enfermos, mostrando un deterioro de la epidermis y del sistema ambulacral, resultando en necrosis epidérmica y la liberación de espinas, dando lugar finalmente a la muerte del individuo. A su vez, al finalizar el experimento y proceder a la disección, los individuos a $17^{\circ} \mathrm{C}$ parecían presentar caparazones frágiles (observación personal). En el erizo Paracentrotus lividus la temperatura afecta el grosor del caparazón y en consecuencia su fragilidad (Byrne \& Przeslawski, 2013). Si bien en el presente trabajo no se realizaron mediciones de dureza y/o grosor, como así tampoco de composición mineral del caparazón, estos parámetros serían de gran importancia para aportar al conocimiento de la fragilidad del caparazón y su relación con el aumento de la temperatura. Por otro lado, el incremento de la temperatura del agua de mar no sólo tiene un efecto directo sobre el organismo, sino uno indirecto dado que también modifica otros factores en su ambiente, como acelerar los procesos bacterianos (White, Kalff, Rasmussen \& Gasol, 1991), favoreciendo las infecciones por bacterias patógenas transmitidas por el agua, dando como resultado eventos de mortalidad (Lessios et al., 1984; Hendler, 2013; Clemente et al., 2014). Probablemente, en este experimento, tanto las heces como los restos de comida hayan producido una mayor carga orgánica, que no haya podido ser totalmente eliminada por los filtros utilizados, y por consiguiente de nutrientes nocivos para los organismos como los compuestos nitrogenados y los fosfatos (Avnimelech, 2006). El incremento de estos compuestos afectan negativamente la fisiología de los organismos, disminuyen las defensas e incrementan la mortalidad de éstos (Böttger, McClintock \& Klinger, 2001; Siikavuopio et al., 2008; Böttger \& McClintock, 2009). Cabe destacar que variables como oxígeno disuelto así como $\mathrm{pH}$ no fueron medidas durante el experimento, estas variables pueden también pueden contribuir en sinergia con la temperatura sobre la mortalidad. De esta forma es difícil poder concluir que la alta mortalidad se debió únicamente a la temperatura del agua.

El incremento del diámetro del caparazón fue mayor en los individuos expuestos al tratamiento térmico de $15{ }^{\circ} \mathrm{C}$ para ambos sexos. En términos absolutos, el incremento fue de 0.022 $\mathrm{mm} / \mathrm{d}$ similar a lo encontrado por Rubilar et al. (2016) en condiciones de laboratorio. Si bien no se observaron diferencias en el incremento del peso total de los erizos de mar expuestos a los diferentes tratamientos, el peso seco de los órganos internos presentó diferencias. Tanto en el peso seco del intestino como en el de las gónadas, se observó un aumento de peso relacionado con el efecto de la temperatura, los valores más altos se observaron en el tratamiento de temperatura intermedia $\left(15^{\circ} \mathrm{C}\right)$. En los erizos de mar, el sistema digestivo es un sistema de órganos conformado por la faringe, el esófago, el sifón, el estómago, el intestino y el ano (Holland, 2013), y comúnmente se llama intestino ("gut", en inglés) al conjunto conformado por el estómago, el intestino y ano; donde el almacenamiento de nutrientes suple la necesidad metabólica a corto plazo (Lawrence, Lawrence, \& Giese, 1966; Fenaux, Malara, Cellario, Charra \& Palazzoli, 1977; Bishop \& Watts, 1992; Hughes, Kelly, Barnes, Catarino, \& Black, 2006). En el intestino de A. dufresnii, se observaron mayores valores de carbohidratos que lípidos en su composición bioquímica evidenciando el rol de almacenamiento de nutrientes (Epherra, 2010). El efecto de la temperatura sobre el peso intestinal se ha observado en Lytechinus variegatus; $S$. intermedius, $P$. lividus y A. lixula, reduciéndose probablemente la capacidad de almacenamiento de nutrientes (Watts et al., 2011; Sastre-Perelló, 2018; Zhao et al., 2018). Por su lado, las gónadas cumplen un doble rol, puesto que generan gametos y almacenan nutrientes a más largo plazo que el intestino (Walker, 1982; Hughes et al., 2006; Walker, Lesser \& Unuma, 2013). En A. dufresnii las gónadas poseen altos valores de proteínas, seguidos por lípidos y en menor medida valores de carbohidratos, evidenciando un rol de almacenamiento (Parra et al., 2015). Para poder analizar el aumento de peso encontrado 
tanto en las gónadas e intestino en este experimento es importante considerar el momento del año en que se realizó el experimento. Durante el otoño, $A$. dufresnii en el sitio estudiado comienza la gametogénesis, la maduración sucede en el invierno y el desove comienza con la primavera y se prolonga durante el verano (Brogger et al., 2010; Epherra et al., 2015), es decir, durante el otoño los erizos de mar invierten gran cantidad de energía en la producción de gametas. El peso de los intestinos a $15{ }^{\circ} \mathrm{C}$ fue similar a los encontrados por Rubilar et al. (2016), posiblemente la alimentación y la asimilación en esta temperatura fue mayor que en los otros tratamientos, pudiendo llevar a cabo ambos procesos metabólicos. Este resultado es relevante dado que una buena condición nutricional en la progenie materna probablemente genere descendencia en mejores condiciones (Byrne et al, 2008).

La gametogénesis de $A$. dufresnii coincide con una disminución de la temperatura del agua de mar en el ambiente natural y el cambio en el fotoperíodo (Brogger et al., 2010; Kino, 2010; Epherra et al., 2015). Al comienzo del experimento, se corroboró que los organismos de ambos sexos se encontraban principalmente en el estadio previo a la gametogénesis, en el estadio pregamético. Los resultados obtenidos indican que tanto la temperatura como el sexo influencian en el peso seco de las gónadas y que estos factores interaccionan entre sí, dado que el peso seco de las gónadas fue mayor $\mathrm{y}$, a su vez, los machos presentaron gónadas de mayor tamaño. La diferencia encontrada entre los sexos es un carácter de esta especie (Brogger et al., 2010; Epherra et al., 2015; Parra et al., 2015; Rubilar et al., 2016; Zárate, Díaz de Vivar, Avaro, Epherra \& Sewell, 2016). Tanto en el diámetro como en el peso seco del intestino, la producción gonadal fue mayor a $15^{\circ} \mathrm{C}$. Paracentrotus lividus presentó un resultado similar, favoreciéndose con un aumento de temperatura intermedio (Santos et al., 2020). Los experimentos a temperaturas elevadas del agua de mar en otros equinoideos han mostrado la disminución de la producción gonadal (Siikavuopio et al., 2008; Delorme \&
Sewell, 2016; Johnstone, Nash, Hernández \& Rahman, 2019; Gouda \& Agatsuma, 2020), lo cual ha sido asociado a una mayor demanda de energía del metabolismo (Uthicke et al., 2014). Experimentos que combinan un aumento de temperatura junto con mayores niveles de $\mathrm{CO}_{2}$ en el agua de mar han demostrado que Echinometra sp. A expuestos a estas condiciones presentan mayor actividad metabólica (medida como respiración), menor peso gonadal y que las gónadas tenían menos gametas maduras (Uthicke et al., 2014).

Las diferencias encontradas entre los tratamientos térmicos están en directa relación con composición celular, es decir, el estadio gonadal. En A. punculata, la producción de gametos de machos y hembras muestra una relación negativa con temperaturas más altas del agua (Johnstone et al., 2019). Mientras que en $P$. lividus, el tratamiento térmico que presentó mayor producción gonadal también promovió los niveles más altos de maduración de las gónadas (Santos et al., 2020). En este trabajo, A. dufresnii mostró que en el tratamiento de temperatura intermedia además de presentar la mayor producción gonadal, los estadios gametogénicos que presentaron fueron similares a los encontrados en la población natural, si bien hay diferencias entre sexos.

Los machos en la población natural, en el mes de julio, se encuentran en estados de crecimiento y premaduración, es decir, que el epitelio germinal está en actividad y que hay presencia de espermatozoides maduros en las gónadas (Epherra et al., 2015). Al comparar los tratamientos, podemos ver que a $13{ }^{\circ} \mathrm{C}$ y 15 ${ }^{\circ} \mathrm{C}$ el escenario es similar. Sin embargo, a 17 ${ }^{\circ} \mathrm{C}$ los individuos también estaban generando gametas y con presencia de espermatozoides pero ya se encontraban individuos desovando, lo cual coincide con los resultados de Uthicke et al. (2014) donde a mayor temperatura las gónadas de los erizos presentan menor cantidad de gametas maduras.

Por su lado, en la población natural las hembras suelen estar en el estadio premaduro y crecimiento al finalizar el experimento (julio). En el experimento, a $13{ }^{\circ} \mathrm{C}$ las gónadas de las 
hembras presentaron variedad de estadios. El $50 \%$ de los individuos estaban en el estadio que se hubiera esperado, crecimiento y premaduro, sin embargo un $30 \%$ se encontraba maduro o desovando y solamente una hembra se encontraba en intergamético. La variabilidad encontrada en hembras a esta temperatura es intrigante, pero a su vez podría relacionarse con el mayor costo energético que requiere la oogenesis (formación de gametas femeninas). Asumiendo que esta temperatura era la más cercana a la promediada para el ambiente natural durante los meses que duró el experimento, se esperaría que sean casi iguales a los estadios encontrados en la población natural, como sucede en machos. Para explicar esta condición se pueden plantear algunas hipótesis. Se sabe que la gametogénesis en hembras es mucho más demandante desde el punto de vista energético que en machos (Walker et al., 2013), que cuando los erizos de mar se encuentran con baja disponibilidad de comida los estadios gonadales tienden a no ser homogéneos entre los individuos, y además de depender de factores como la temperatura y el fotoperiodo, también dependen de sus propias reservas energéticas (Lawrence, Lawrence \& Watts, 2013; Rubilar et al., 2016). Por lo tanto, es posible que las hembras no hayan ingerido comida suficiente, dado que la temperatura es el factor que más afecta el consumo de alimento y por lo tanto la energía obtenida (James, Siikavuopio \& Mortensen, 2015). En Strongylocentrotus droebachiensis la tasa de alimentación aumenta con la temperatura casi en una relación lineal en experimentos de laboratorio (Siikavuopio, Christiansen \& Dale, 2006). En cambio, en el ambiente natural, la relación no es lineal pero está fuertemente relacionada a la temperatura y el ciclo reproductivo que depende del fotoperiodo (Scheibling \& Hatcher, 2007). Durante el experimento, las hembras encontradas en estadios de desove comieron la mitad de las veces que lo hicieron las hembras en estadio de crecimiento, premaduro y maduro; y a su vez, el individuo en estadio intergamético comió una sola vez durante todo el experimento. Probablemente, la menor ingestión de alimento podría ser la causa de la composición celular de las gónadas las cuales no se encontraron en crecimiento. Sin embargo, los vínculos subyacentes entre la ingesta de alimento y el ciclo reproductivo aún no están claros (James et al., 2015). Estudios de laboratorio mostraron una completa falta de respuesta en la ingesta de alimento por parte de los erizos de mar cuando se exponen a condiciones de mantenimiento subóptimas (Siikavuopio, 2009). A todos los individuos del experimento se les ofreció alimento ad libitum, de esta forma los resultados observados en hembras en la temperatura 13 ${ }^{\circ} \mathrm{C}$ pudiesen atribuírse a condiciones de mantenimiento subóptimas. Por su lado, en el tratamiento de $17^{\circ} \mathrm{C}$ las hembras se comportaron de forma análoga a los machos; y a $15^{\circ} \mathrm{C}$, en cambio, ya hay presencia de individuos maduros y desove, aún no registrado en el ambiente natural para este periodo otoñal.

Como conclusión de este estudio, se encontró que en el tratamiento de $15^{\circ} \mathrm{C}$, los individuos de $A$. dufresnii, presentaron cambios favorables en el incremento en el diámetro, producción gonadal así como también un aumento en el peso del intestino a pesar de registrarse una relativa alta mortalidad. De los tres tratamientos ensayados, esta es la temperatura óptima para el desarrollo y crecimiento de estos organismos en las condiciones experimentales probadas, si bien presentó una mortalidad mayor que a $13{ }^{\circ} \mathrm{C}$.

Finalmente, si bien el océano irá cambiando más gradualmente su temperatura durante las próximas décadas, es probable que muchas especies puedan tolerar cambios futuros mediante la aclimatación o la adaptación. Sin embargo, existen pocos estudios de tolerancia térmica en donde se evalúe a los erizos de mar adultos en el contexto del cambio climático global. Por lo tanto, el presente trabajo permitiría sentar las bases de los efectos de la temperatura en la población de $A$. dufresnii en el Golfo Nuevo. Por otra parte, se requeriría de más trabajos donde se consideren los efectos de las interacciones sinérgicas entre muchos factores antropogénicos, como la mayor radiación UV (debido a la ruptura de la capa de ozono), la 
contaminación y la acidificación de los océanos para predecir mejor los cambios futuros en la estructura de los ecosistemas. Para comprender completamente las consecuencias biológicas del cambio climático, múltiples variables climáticas deben ser manipuladas experimentalmente en conjunto.

Declaración de ética: los autores declaran que todos están de acuerdo con esta publicación y que han hecho aportes que justifican su autoría; que no hay conflicto de interés de ningún tipo; y que han cumplido con todos los requisitos y procedimientos éticos y legales pertinentes. Todas las fuentes de financiamiento se detallan plena y claramente en la sección de agradecimientos. El respectivo documento legal firmado se encuentra en los archivos de la revista.

\section{AGRADECIMIENTOS}

\author{
Este trabajo fue financiado por el Subsidio \\ de CONICET PICT 2016-0231.
}

\section{RESUMEN}

Introducción: El aumento de los valores del $\mathrm{CO}_{2}$ atmosférico y el consecuente calentamiento del agua de mar constituyen factores de estrés para los ecosistemas marinos, renovando el interés en la fisiología de los equinoideos. El equinoideo Arbacia dufresnii es una especie ampliamente estudiada y con amplia distribución en los golfos norpatagónicos. Objetivo: Evaluar el efecto de temperatura del agua de mar sobre su morfología y reproducción. Métodos: Individuos de $A$. dufresnii fueron expuestos durante 10 semanas (Abril-Junio 2016) a tres tratamientos de temperatura $\left(13^{\circ} \mathrm{C}\right.$ control, $15^{\circ} \mathrm{C}$ y $\left.17^{\circ} \mathrm{C}\right)$ de acuerdo con las condiciones actuales del agua de mar y los valores esperados para el próximo siglo, según diferentes escenarios estimados. Al finalizar el experimento, diámetro y peso del individuo así como los pesos de los órganos internos fueron estadística e histológicamente analizados. Resultados: Se observó una mayor mortalidad en los individuos expuestos a las mayores temperaturas lo que podría deberse a un estrés en el individuo. Para el tratamiento control y el de $17^{\circ} \mathrm{C}$ no se observaron cambios en los pesos secos de gónadas e intestino. En cambio, los individuos expuestos a $15{ }^{\circ} \mathrm{C}$ presentaron un aumento en el diámetro del caparazón y en el peso seco de gónadas e intestino. Los estadios gonadales aesta temperatura $\left(15^{\circ} \mathrm{C}\right)$ para machos fueron similares al ambiente a diferencia de las hembras que se observaron estadios más avanzados. Conclusiones: Esto indicaría que $15{ }^{\circ} \mathrm{C}$ podría ser la temperatura óptima para la asimilación del alimento y obtención de energía. A esta temperatura se observan cambios a corto plazo en los órganos de metabolismo rápido pero no en los órganos calcificados (caparazón y linterna de Aristóteles).

Palabras clave: equinoideos; experimento térmico; gametogénesis; producción gonadal; fisiología

\section{REFERENCIAS}

Avnimelech, Y. (2006). Bio-filters: the need for a new comprehensive approach. Aquacultural Engineering, 34(3), 172-178.

Azad, A.K., Pearce, C.M. \& McKinley, R.S. (2011). Effects of diet and temperature on ingestion, absorption, assimilation, gonad yield, and gonad quality of the purple sea urchin (Strongylocentrotus purpuratus). Aquaculture, 317(1), 187-196.

Bates, W.R. (2005). Environmental factors affecting reproduction and development in ascidians and other protochordates. Canadian Journal of Zoology, 83(1), 51-61.

Bernasconi, I. (1953). Monografía de los Equinoideos argentinos. Anales del Museo de Historia Natural, $6(2), 1-58$.

Bishop, C. \& Watts, S. (1992). Biochemical and morphometric study of growth in the stomach and intestine of the echinoid Lytechinus variegatus (Echinodermata). Marine Biology, 114(3), 459-467.

Böttger, S.A. \& McClintock, J.B. (2009). The effects of chronic inorganic and organic phosphate exposure on bactericidal activity of the coelomic fluid of the sea urchin Lytechinus variegatus (Lamarck) (Echinodermata: Echinoidea). Comparative Biochemistry and Physiology Part C: Toxicology \& Pharmacology, 150(1), 39-44.

Böttger, S., McClintock, J. \& Klinger, T. (2001). Effects of inorganic and organic phosphates on feeding, feeding absorption, nutrient allocation, growth and righting responses of the sea urchin Lytechinus variegatus. Marine Biology, 138(4), 741-751.

Brierley, A.S. \& Kingsford, M.J. (2009). Impacts of climate change on marine organisms and ecosystems. Current Biology, 19(14), 602-614.

Brogger, M., Martinez, M. \& Penchaszadeh, P. (2010). Reproduction of the sea urchin Arbacia dufresnii (Echinoidea: Arbaciidae) from Golfo Nuevo, Argentina. Journal of the Marine Biological Association of the United Kingdom, 90(7), 1405-1409.

Brogger, M., Gil, D.G., Rubilar, T., Martínez, M.I., Díaz de Vivar, M.E., Escolar, M., . . . Tablado, A. (2013). 
Echinoderms from Argentina: Biodiversity, distribution and current state of knowledge. In J.J. Alvarado \& F.A. Solís-Marin (Eds.), Echinoderm Research and Diversity in Latin America (pp. 359-402). Berlín: Springer Heidelberg.

Byrne, M. (2010). Impact of climate change stressors on marine invertebrate life histories with a focus on the Mollusca and Echinodermata. In J. Yu \& A. Henderson (Eds.), Climate Alert: Climate Change Monitoring and Strategy (pp. 142-185). Sydney: University of Sydney Press.

Byrne, M., Andrew, N., Worthington, D. \& Brett, P. (1998). Reproduction in the diadematoid sea urchin Centrostephanus rodgersii in contrasting habitats along the coast of New South Wales, Australia. Marine Biology, 132(2), 305-318.

Byrne, M., Prowse, T.A.A., Sewell, M.A., Dworjanyn, S.A., Williamson, J.E. \& Vaitilington, D. (2008) Maternal provisioning for larvae and larval provisioning for juveniles in the toxopneustid sea urchin Tripneustes gratilla. Marine Biology; 55, 473-482.

Byrne, M., Ho, M., Selvakumaraswamy, P., Nguyen, H.D., Dworjanyn, S.A. \& Davis, A.R. (2009). Temperature, but not $\mathrm{pH}$, compromises sea urchin fertilization and early development under near-future climate change scenarios. Proceedings of the Royal Society of London B: Biological Sciences, 276(1663), 1883-1888.

Byrne, M. \& Przeslawski, R. (2013). Multistressor impacts of warming and acidification of the ocean on marine invertebrate life histories. Integrative and Comparative Biology, 53(4), 582-596.

Clemente, S., Lorenzo-Morales, J., Mendoza, J., López, C., Sangil, C., Alves, F., . . . Hernández, J. (2014). Sea urchin Diadema africanum mass mortality in the subtropical eastern Atlantic: role of waterborne bacteria in a warming ocean. Marine Ecology Progress Series, 506, 1-14.

Cohen-Rengifo, M., García, E., Hernández, C.A., Hernández, J.C. \& Clemente, S. (2013). Global warming and ocean acidification affect fertilization and early development of the sea urchin Paracentrotus lividus. Cahiers de Biologie Marine, 54, 667-675.

Delorme, N.J. \& Sewell, M.A. (2016). Effects of warm acclimation on physiology and gonad development in the sea urchin Evechinus chloroticus. Comparative Biochemistry and Physiology Part A: Molecular \& Integrative Physiology, 198, 33-40.

Dellatorre, F.G., Pisoni, J.P., Barón, P.J. \& Rivas, A.L. (2012). Tide and wind forced nearshore dynamics in Nuevo Gulf (Northern Patagonia, Argentina): Potential implications for cross-shore transport. Journal of Marine Systems, 96, 82-89.

Dupont, S. \& Thorndyke, M. (2009). Impact of $\mathrm{CO}_{2}$ driven ocean acidification on invertebrate early
life-history-What we know, what we need to know and what we can do. Biogeosciences Discussions, 6(2), 3109-3131.

Ebert, T., Hernandez, J. \& Russell, M. (2011). Problems of the gonad index and what can be done: analysis of the purple sea urchin Strongylocentrotus purpuratus. Marine Biology, 158(1), 47-58.

Edwards, M. \& Richardson, A.J. (2004). Impact of climate change on marine pelagic phenology and trophic mismatch. Nature, 430(7002), 881.

Epherra, L. (2010). Ciclo bioquímico del erizo verde en costas del Golfo Nuevo, Patagonia. (Tesis de licenciatura). Universidad Nacional de la Patagonia San Juan Bosco, Argentina.

Epherra, L. (2016). Evaluación del impacto de invertebrados herbivoros nativos sobre el alga invasora Undaria pinnatifida: Arbacia dufresnii (Echinodermata: Echinoidea) como modelo de estudio. (Tesis de doctorado). Universidad Nacional de Mar del Plata, Argentina.

Epherra, L., Gil, D.G., Rubilar, T., Pérez-Gallo, S., Reartes, M.B. \& Tolosano, J.A. (2015). Temporal and spatial differences in the reproductive biology of the sea urchin Arbacia dufresnii. Marine and Freshwater Research, 66(4), 329-342.

Fenaux, L., Malara, G., Cellario, C., Charra, R. \& Palazzoli, I. (1977). Evolution des constituants biochimiques des principaux compartiments de l'oursin Arbacia lixula (L.) au cours d'un cycle sexuel et effets d'un jeûne de courte durée au cours de la maturation sexuelle. Journal of Experimental Marine Biology and Ecology, 28(1), 17-30.

Fernández, J.P., Chaar, F.B., Epherra, L., González-Aravena, J.M., \& Rubilar, T. (2021). Embryonic and larval development is conditioned by water temperature and maternal origin of eggs in the sea urchin Arbacia dufresnii (Echinodermata: Echinoidea). Revista de Biología Tropical, 69(S1), 452-463. DOI 10.15517/ rbt.v69iSupp1.1.46384.

González, R.A., Díaz, F., Licea, A., Re, A.D., Sánchez, L.N. \& García-Esquivel, Z. (2010). Thermal preference, tolerance and oxygen consumption of adult white shrimp Litopenaeus vannamei (Boone) exposed to different acclimation temperatures. Journal of Thermal Biology, 35(5), 218-224.

Gooding, R.A., Harley, C.D. \& Tang, E. (2009). Elevated water temperature and carbon dioxide concentration increase the growth of a keystone echinoderm. Proceedings of the National Academy of Sciences, 106(23), 9316-9321.

Gouda, H. \& Agatsuma, Y. (2020). Effect of high temperature on gametogenesis of the sea urchin Strongylocentrotus intermedius in the Sea of Japan, northern 
Hokkaido, Japan. Journal of Experimental Marine Biology and Ecology, 525, 151324.

Harianto, J., Nguyen, H.D., Holmes, S.P. \& Byrne, M. (2018). The effect of warming on mortality, metabolic rate, heat-shock protein response and gonad growth in thermally acclimated sea urchins (Heliocidaris erythrogramma). Marine Biology, 165(6), 96.

Harrold, C. \& Pearse, J.S. (1987). The ecological role of echinoderms in kelp forests. Echinoderm studies, 2, 137-233.

Hendler, G. (2013). Recent mass mortality of Strongylocentrotus purpuratus (Echinodermata: Echinoidea) at Malibu and a review of purple sea urchin kills elsewhere in California. Bulletin, Southern California Academy of Sciences, 112(1), 19-37.

Hernández, M., Bückle, F., Guisado, C., Barón, B. \& Estavillo, N. (2004). Critical thermal maximum and osmotic pressure of the red sea urchin Strongylocentrotus franciscanus acclimated at different temperatures. Journal of Thermal Biology, 29(4-5), 231-236.

Hochachka, P. \& Somero, G. (2002). Biochemical adaptation, mechanism and process in physiological evolution. New York, USA: Oxford University Press.

Holland, N.D. (2013). Digestive system Developments in Aquaculture and Fisheries Science (Vol. 38). Ciudad, País: Elsevier.

Hughes, A.D., Kelly, M.S., Barnes, D.K., Catarino, A.I. \& Black, K.D. (2006). The dual functions of sea urchin gonads are reflected in the temporal variations of their biochemistry. Marine Biology, 148(4), 789-798.

IPCC. (2013). Climate Change 2013: The Physical Science Basis. Contribution of Working Group I to the Fifth Assessment Report of the Intergovernmental Panel on Climate Change. T.F. Stocker et al. (Eds.). United Kingdom, New York USA: Cambridge University Press.

IPCC. (2014). Climate change 2014: Synthesis Report. Contribution of Working Groups I, II and III to the fifth assessment report of the Intergovernmental Panel on Climate Change. R.K. Pachauri \& L.A. Meyer (Eds.). Geneva, Switzerland: IPCC.

James, P., Siikavuopio, S. \& Mortensen, A. (2015). Sea urchin aquaculture in Norway. In N.P Brown, S.D. (Eds.), Echinoderm Aquaculture (pp. 147-173). New York, USA: Wiley.

Johnstone, J., Nash, S., Hernandez, E. \& Rahman, M.S. (2019). Effects of elevated temperature on gonadal functions, cellular apoptosis, and oxidative stress in Atlantic sea urchin Arbacia punculata. Marine Environmental Research, 149, 40-49.

Kemp, J. \& Britz, P. (2008). The effect of temperature on the growth, survival and food consumption of the east coast rock lobster Panulirus homarus rubellus. Aquaculture, 280(1), 227-231.

Kino, S. (2010). Reproduction and early life history of sea urchins, Arbacia dufresnei and Pseudechinus magellanicus, in Chiloé Island and Reloncaví Sound, Chile. Aquaculture Science, 58(1), 65-73.

Kurihara, H. \& Shirayama, Y. (2004). Effects of increased atmospheric $\mathrm{CO}_{2}$ on sea urchin early development. Marine Ecology Progress Series, 274, 161-169.

Lawrence, A.J. \& Soame, J.M. (2004). The effects of climate change on the reproduction of coastal invertebrates. Ibis, 146, 29-39.

Lawrence, J.M. \& Ellwood, A. (1991). Simultaneous allocation of resources to arm regeneration and to somatic and gonadal production in Luidia clathrata (Say) (Echinodermata: Asteroidea). In T. Yanagisawa, T., I. Yasumasu, C. Oguro, N. Suzuki \& T. Motokawa, (Eds), Biology of Echinodermata (pp. 543-548). Netherlands: Balkema.

Lawrence, J.M., Lawrence, A. \& Giese, A. (1966). Role of the gut as a nutrient-storage organ in the purple sea urchin (Strongylocentrotus purpuratus). Physiological Zoology, 39(4), 281-290.

Lawrence, J.M., Cao, X., Chang, Y., Wang, P., Yu, Y., Lawrence, A.L. \& Watts, S.A. (2009). Temperature effect on feed consumption, absorption, and assimilation efficiencies and production of the sea urchin Strongylocentrotus intermedius. Journal of Shellfish Research, 28(2), 389-395.

Lawrence, J.M., Lawrence, A.L. \& Watts, S.A. (2013). Feeding, Digestion and Digestibility of Sea Urchins. In J.M: Lawrence (Ed), Sea Urchins: Biology and Ecology (pp. 135-150). Amsterdam: Elsevier Science.

Lessios, H., Cubit, J., Robertson, D., Shulman, M., Parker, M., Garrity, S. \& Levings, S. (1984). Mass mortality of Diadema antillarum on the Caribbean coast of Panama. Coral Reefs, 3(4), 173-182.

McCullagh, P. (1984). Generalized linear models. European Journal of Operational Research, 16(3), 285-292.

McElroy, D., Nguyen, H. \& Byrne, M. (2012). Respiratory response of the intertidal seastar Parvulastra exigua to contemporary and near-future pulses of warming and hypercapnia. Journal of Experimental Marine Biology and Ecology, 416, 1-7.

McGaw, I.J., Clifford, A.M. \& Goss, G.G. (2015). Physiological responses of the intertidal starfish Pisaster ochraceus, (Brandt, 1835) to emersion at different temperatures. Journal of Experimental Marine Biology and Ecology, 468, 83-90.

Olive, P. (1995). Annual breeding cycles in marine invertebrates and environmental temperature: probing the proximate and ultimate causes of reproductive 
synchrony. Journal of Thermal Biology, 20(1-2), 79-90.

Paine, R.T. (1966). Food web complexity and species diversity. The American Naturalist, 100(910), 65-75.

Parra, M., Rubilar, T., Latorre, M., Epherra, L., Gil, D.G. \& Díaz de Vivar, M.E. (2015). Nutrient allocation in the gonads of the sea urchin Arbacia dufresnii in different stages of gonadal development. Invertebrate Reproduction \& Development, 59(1), 26-36.

Pérez, A.F., Gil, D.G. \& Rubilar, T. (2014). Echinodermata. In J.A. Calcagno (Ed.), Los invertebrados Marinos (pp. 295-316). Buenos Aires, Argentina: Vazquez Mazzini.

Pisoni, J., Dellatorre, F. \& Rivas, A. (2017). Temperatura del mar en la estación oceanográfica muelle Luis Piedrabuena durante el periodo 2011-2016. Argentina: Centro Nacional Patagónico. Informe técnico.

Przeslawski, R., Ahyong, S., Byrne, M., Woerheide, G. \& Hutchings, P. (2008). Beyond corals and fish: the effects of climate change on noncoral benthic invertebrates of tropical reefs. Global Change Biology, 14(12), 2773-2795.

R Core Team. (2014). $R$ : A language and environment for statistical computing. R Foundation for Statistical Computing, Vienna, Austria. Retrieved from

Rubilar, T. \& Crespi-Abril, A. (2017). Does Echinoderm research deserve an ethical consideration? Revista de Biología Tropical, 65(1), 11-22.

Rubilar, T., Epherra, L., Deias-Spreng, J., Díaz de Vivar, M.E., Avaro, M., Lawrence, A. \& Lawrence, J. (2016). Ingestion, absorption and assimilation efficiencies, and production in the sea urchin Arbacia dufresnii fed a formulated feed. Journal of Shellfish Research, 35, 1083-1093.

Russell, M.P. (1998). Resource allocation plasticity in sea urchins: rapid, diet induced, phenotypic changes in the green sea urchin, Strongylocentrotus droebachiensis (Müller). Journal of Experimental Marine Biology and Ecology, 220(1), 1-14.

Sanford, E. (2002). Water temperature, predation, and the neglected role of physiological rate effects in rocky intertidal communities. Integrative and Comparative Biology, 42(4), 881-891.

Santos, P.M., Albano, P., Raposo, A., Ferreira, S.M., Costa, J.L. \& Pombo, A. (2020). The effect of temperature on somatic and gonadal development of the sea urchin Paracentrotus lividus (Lamarck, 1816). Aquaculture, 528,735487 .

Sastre-Perelló, M. (2018). Influencia de la temperatura en la ecología de los herbívoros invertebrados dominantes del litoral mediterráneo (Paracentrotus lividus y
Arbacia lixula) (Tesis de maestría). Universitat de les Illes Balears, España.

Scheibling, R. \& Hatcher, B. (2007). Ecology of Strongylocentrotus droebachiensis. In J.M Lawrence (Ed.), Developments in Aquaculture and Fisheries Science (Vol. 37, pp. 353-392). Amsterdam: Elsevier Science BV.

Siikavuopio, S.I. (2009). Green sea urchin (Strongylocentrotus droebachiensis, Müller) in aquaculture: the effects of environmental factors on gonad growth. (Doctoral dissertation). University of Tromsø. Norway.

Siikavuopio, S.I., Christiansen, J.S. \& Dale, T. (2006). Effects of temperature and season on gonad growth and feed intake in the green sea urchin (Strongylocentrotus droebachiensis). Aquaculture, 255(1-4), 389-394.

Siikavuopio, S.I., Mortensen, A. \& Christiansen, J.S. (2008). Effects of body weight and temperature on feed intake, gonad growth and oxygen consumption in green sea urchin, Strongylocentrotus droebachiensis. Aquaculture, 281(1), 77-82.

Siikavuopio, S.I., James, P., Lysne, H., Sæther, B.S., Samuelsen, T.A. \& Mortensen, A. (2012). Effects of size and temperature on growth and feed conversion of juvenile green sea urchin (Strongylocentrotus droebachiensis). Aquaculture, 354, 27-30.

Uthicke, S., Liddy, M., Nguyen, H. \& Byrne, M. (2014). Interactive effects of near-future temperature increase and ocean acidification on physiology and gonad development in adult Pacific sea urchin, Echinometra sp. Coral Reefs, 33(3), 831-845.

Walker, C.W. (1982). Nutrition of gametes. In M. Jangoux \& J.M: Lawrence (Eds), Echinoderm nutrition (pp. 449-468). Netherlands: Balkema.

Walker, C.W., Lesser, M. \& Unuma, T. (2013). Sea urchin gametogenesis-structural, functional and molecular/ genomic biology. In J.M: Lawrence (Ed), Sea Urchins: Biology and Ecology (pp. 25-43). Amsterdam: Elsevier Science.

Wangensteen, O.S., Turon, X., Casso, M. \& Palacín, C. (2013). The reproductive cycle of the sea urchin Arbacia lixula in northwest Mediterranean: potential influence of temperature and photoperiod. Marine Biology, 160(12), 3157-3168.

Watts, S.A., Hofer, S.C., Desmond, R.A., Lawrence, A.L. \& Lawrence, J.M. (2011). The effect of temperature on feeding and growth characteristics of the sea urchin Lytechinus variegatus fed a formulated feed. Journal of Experimental Marine Biology and Ecology, 397(2), 188-195.

White, P.A., Kalff, J., Rasmussen, J.B. \& Gasol, J.M. (1991). The effect of temperature and algal biomass 
on bacterial production and specific growth rate in freshwater and marine habitats. Microbial ecology, 21(1), 99-118.

Wilson, I. \& Gamble, M. (2002). The hematoxylins and eosin. In J. Bancroft \& M. Gamble (Eds.), Theory and practice of histological techniques (pp. 125-138). London, United Kingdom: Elsevier.

Zamora, L.N. \& Jeffs, A.G. (2012). Feeding, metabolism and growth in response to temperature in juveniles of the Australasian sea cucumber, Australostichopus mollis. Aquaculture, 358, 92-97.
Zárate, E.V., Díaz de Vivar, M.E., Avaro, M.G., Epherra, L. \& Sewell, M.A. (2016). Sex and reproductive cycle affect lipid and fatty acid profiles of gonads of Arbacia dufresnii (Echinodermata: Echinoidea). Marine Ecology Progress Series, 551, 185-199.

Zhao, C., Zhang, L., Qi, S., Shi, D., Yin, D. \& Chang, Y. (2018). Multilevel effects of long-term elevated temperature on fitness related traits of the sea urchin Strongylocentrotus intermedius. Bulletin of Marine Science, 94(4), 1483-1497. 